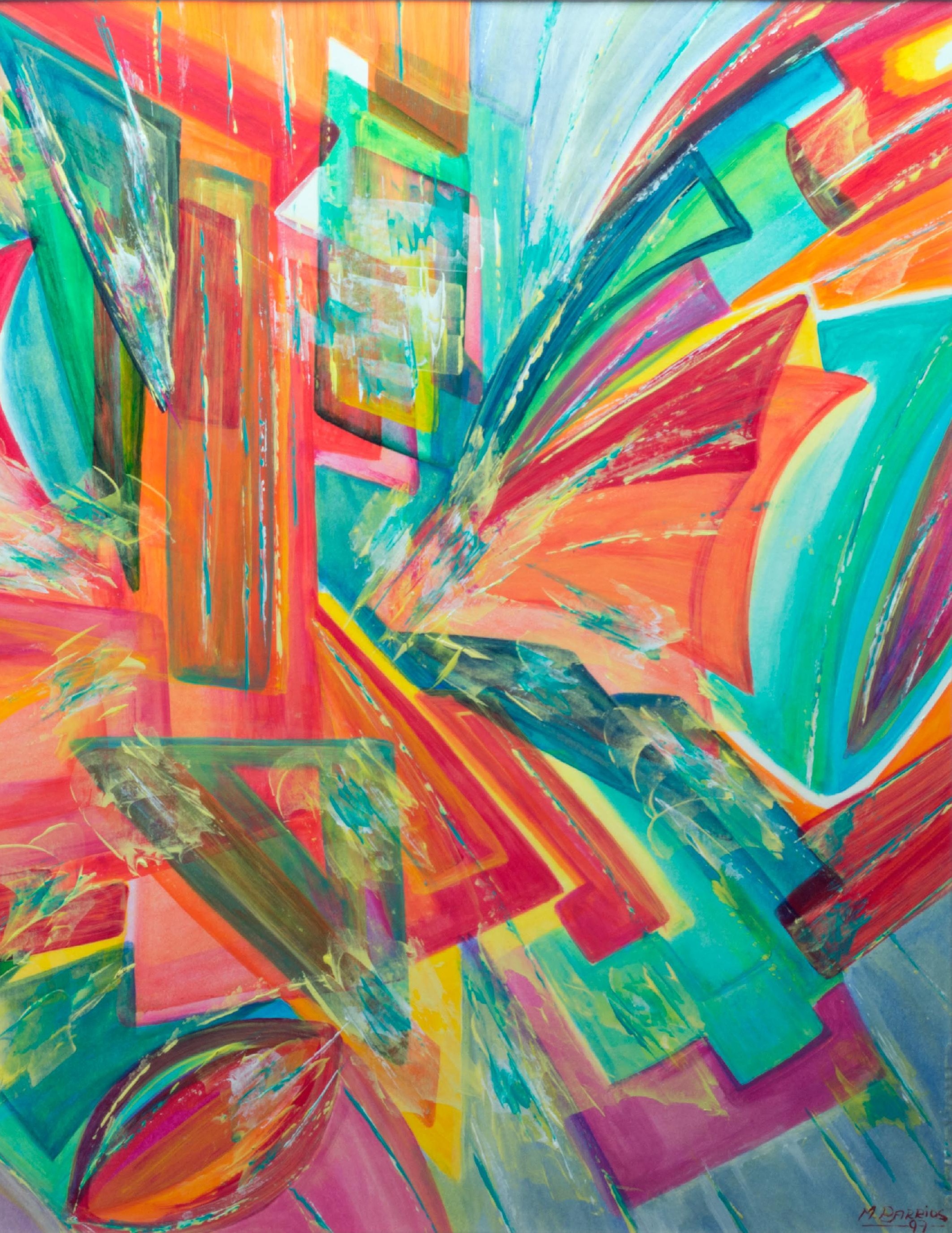




\section{cMaldita enfermedad}

Injusta,

Por definición siempre es,

Destino de algunos que nadie merece.

Intempestiva y traicionera,

Ladrona sigilosa de la vida al entrar sin siquiera llamar.

Dolorosa que ciencia todavía no alcanza a curar,

$\mathcal{N}$ i tan siquiera con placebos aliviar.

Tirana con la que luchar,

Mantener costumbres y rutinas,

Con inmenso esfuerzo adicional.

Asesina de sueños, de la esperanza de sanar,

Materia que al final no es más que mortal.

\section{Invasora de hogares,}

Catadora incesante de la fuerza y resistencia de sus pilares.

Torturadora que carcome la energía vital

Yel alma desangra, gota a gota, día a día, siempre algo más.

Insidiosa que contagia, sin contagiar,

Únicamente espíritu de resistencia sin otras armas para batallar.

Imborrable marcadora de surcos imposibles de desdibujar,

Del que estoicamente soporta,

$Y$ de aquel que con locura impotente acompaña el malestar.

Maldita enfermedad,

Es su lento caminar viento huracanado que asola todo al pasar,

La sonrisa, pasión, alegría y, en ocasiones,

Hasta el deseo de respirar.

8003

Marta Doreste 


\title{
7. Mortalidad proporcional en tres años, Hospital San Felipe, M.D.C.
}

\author{
María Guadalupe Romero ${ }^{1}$ e Ingrid Carolina Garay² \\ Recibido 31-07-2017 / Aceptado 23-08-2017
}

\begin{abstract}
RESUMEN: Existe una serie de indicadores que son utilizados para medir las condiciones de salud de una población.Al implementarse la estrategia de Salud para Todos en los años 80 's, se planteó el uso de 12 indicadores entre los que se encontraba el de mortalidad, el que es un indicador de resultado y es un excelente instrumento de trabajo para las autoridades de los países en vías de desarrollo. Basado en lo anterior, el presente estudio es de diseño transversal y retrospectivo, incluye datos de tres años, 20I 4, 2015 y 20I6, haciendo énfasis en la mortalidad proporcional por causa, siendo la primera causa de muerte, el cáncer, la segunda causa, la septicemia y las siguientes causas de muerte están todas relacionadas con las enfermedades no transmisibles. Siendo un hospital nacional de referencia que incluye el servicio de maternidad, también se incluye como la última causa de muerte la relacionada con afecciones que atañen al grupo menores de un año de edad. Cada una de estas causas están relacionadas con factores de riesgo específicos que se deben tomar en cuenta para la planificación de programas de prevención sobre todo en el análisis de la información que posteriormente a la revisión de los mismos permite conocer el comportamiento en los años expuestos, razón por la cual deben ser tomados en cuenta para la planificación de los servicios del hospital y de toda la red hospitalaria del país. La mortalidad hospitalaria aún con la limitación de ser un indicador negativo de salud, constituye un aspecto muy importante en la investigación clínica y epidemiológica y en el conocimiento de los problemas de salud de la población que acude al hospital.
\end{abstract}

Palabras clave: mortalidad, mortalidad proporcional, causa básica de muerte.

ABSTRACT: There are a number of indicators that are used to measure the health conditions of a population. When implementing the Health for All strategy in the 1980s, the use of 12 indicators, including mortality, was considered, which is an outcome indicator and is an excellent working tool for health authorities. developing countries. Based on the above, the present study is cross-sectional and retrospective, including data for three years, 2014,2015 and 2016, with emphasis on proportional mortality by cause, being the leading cause of death, cancer, the second cause, Septicemia and the following causes of death are all related to noncommunicable diseases. Being a national referral hospital that includes maternity service, it is also included as the last cause of death related to affections that affect the group under one year of age. Each of these causes are related to specific risk factors that must be taken into account for the planning of prevention programs, especially in the analysis of the information that after the review of the same allows to know the behavior in the exposed years, Which is why they should be taken into account for the planning of hospital services and the entire hospital network in the country. Hospital mortality, with the limitation of being a negative indicator of health, is a very important aspect in clinical and epidemiological research and in the knowledge of the health problems of the population that goes to the hospital.

Keywords: mortality, proportional mortality, basic cause of death.

\section{Introducción}

De todas las actividades que se generan en un hospital, las estadísticas hospitalarias son las que han experimentado un gran avance en los últimos años. Este hecho se explica por la cuantiosa información que se puede obtener de cada institución hospitalaria.

Desde los años 1994-1995, dando respuesta a los procesos de descentralización y para lograr apoyar a las nuevas funciones y responsabilidades de los diferentes niveles de servicios de salud, la OPS reconoció la importancia de disponer de datos e indicadores sobre la situación de salud para orientar la ejecución de los programas de cooperación técnica y estimuló la conformación de datos básicos como un conjunto integral de indicadores esenciales, relacionados con la salud, para caracterizar en forma cuantitativa la situación de un país o de una región (OPS,
Evaluación decenal de la Iniciativa Regional de Datos Básicos de Salud, 2004).

La utilidad de las estadísticas de mortalidad como indicadores de salud, a pesar de sus limitaciones, es insustituible. La responsabilidad médica en la consignación de las causas de defunción constituye uno de los factores más fácilmente mejorables.

Los estudios de mortalidad proporcional constituyen un tipo particular de diseño incompleto en el que la información sobre el tamaño de la población a riesgo ( o población- tiempo expuesta) de la que surgieron los casos de muerte, no es conocida. Se han empleado en epidemiología partiendo de la información contenida en los certificados de defunción. Desde Miettinen y Wang en 1981, se insiste puede ser considerado un tipo especial de estudio transversal en el que en forma típica, se comparan

\footnotetext{
${ }^{1}$ Médico y Cirujano. Máster en Salud Pública, Máster en Administración de Servicios de Salud. PhD. mgromeroa@yahoo.com

${ }^{2}$ Ingeniera en Ciencias Ambientales, Máster en Ciencias, Tecnología y Gestión del Agua, PhD. icgaray@usal.es
} 
los sujetos que han muerto por la enfermedad objeto de investigación frente a una muestra de los sujetos que han fallecido por otras causas durante el mismo período de tiempo (Delgado R, Sillero, \& Galvez, 1994). La mortalidad es un indicador esencial para estudiar la evolución de ésta y de su significado sobre la salud, y se ha considerado como un indicador negativo de salud. Sin embargo, el campo de la aplicación potencial de estas estadísticas es mucho más amplio, de tal forma que, al disponer de ellas con puntualidad, se pueden utilizar como señales de alerta. Cabe destacar que desde que David Rutstein en 1976 se preguntara al observar los datos de mortalidad de un hospital ante una muerte de causa dudosa, se mantiene la necesidad de conocer las causas que desencadenan la muerte en enfermedades que no debieran terminar fatalmente en ese momento. Analizar las características clínicas de los fallecidos, así como las causas de muerte, permite conocer no solo la estadística de mortalidad hospitalaria sino también si la muerte era evitable para evitar su repetición permitiendo apreciar la evolución de los datos (Revista, 2013).

La prevalencia de estas causas, no pueden extrapolarse a las poblaciones, a menos que, solo se atiendan a pobladores cautivos por su residencia o pertenencia a una determinada comunidad. Del mismo modo, no pueden extrapolarse a otros establecimientos aunque aparentemente sean similares en complejidad porque la afluencia de internados dependerá de la complejidad y del peso de la composición de los distintos servicios de la institución o por ser centros de derivación de determinada enfermedad o riesgo.

Las diferencias observadas en la mortalidad por causas, de acuerdo a las distintas características de las personas que mueren, v.g. edad y sexo o del tiempo (año), permiten detectar la presencia de factores de riesgo y su efecto. La muerte, es un hecho inevitable, razón por la cual, Rutsein en 1976, propuso su vigilancia como indicador de calidad de los servicios médicos y sanitarios (Segura, 1989).

A partir de esa propuesta, la muerte es un indicador bruto que se encuentra influenciado por numerosos factores entre los que cabe, patología atendida, estructura etárea de la población, régimen económico del centro asistencial y accesibilidad a los hospitales.

La mortalidad hospitalaria, con las limitaciones propias de ser un indicador negativo, constituye un instrumento imprescindible en la investigación clínica y epidemiológica. Asimismo, es fundamental en la evaluación de los programas de garantía de calidad y en el conocimiento de los problemas de salud de la población del área de influencia del hospital (García Ortega, Almenara Barrios, \& García Ortega, 1997).

Revista Población y Desarrollo: argonautas y caminantes
La supervisión de los datos estadísticos y el análisis crítico de la mortalidad hospitalaria son útiles para determinar aquellas causas que pudieron haber sido evitadas y valiosas herramientas para la planificación y la gestión. La cuantificación de las defunciones puede considerarse como una medida de efectividad de la intervención hospitalaria (Revista, 2013).

El presente trabajo realiza una revisión de las muertes hospitalarias ocurridas en los años 2014, 2015 y 2016 en el hospital San Felipe de Tegucigalpa, siendo este un centro de referencia nacional en el cual se realiza una descripción de la causa básica de muerte por año a través de la revisión de la mortalidad proporcional y la distribución de la misma en los años referidos.

\section{Metodología y aspectos conceptuales}

Se trata de un estudio descriptivo, transversal y retrospectivo en el cual se recogió la información de las defunciones (muertes básicas) acaecidas en los años 2014, 2015 y 2016 en el hospital San Felipe de Tegucigalpa. Los datos fueron tomados de los expedientes clínicos de los pacientes que fallecieron en los períodos señalados, haciéndose revisión de la hoja de egreso, del certificado de defunción y la epicrisis. Asimismo, se incluyeron, datos de sexo, edad y causa primaria de muerte (de acuerdo a Clasificación Internacional 10). En esta investigación, se toma la causa básica de muerte que aparece en la hoja de egreso.

Con los datos señalados, se construyeron tablas de frecuencia de la variable sexo, edad (datos no agrupados) y descripción de medidas de tendencia central y de desviación, partiendo del menor de 1 año hasta la edad de 95 años, realizando el cálculo con el paquete SPSS 23.

Para el cálculo de mortalidad proporcional por causa, se utiliza la fórmula del cociente del número de defunciones por determinada causa entre el total de los muertos en un año (http://publications. paho.org/spanish/PC+629+Cap_2.pdf) (Cuba, 2010).

La estadística hospitalaria puede utilizarse fundamentalmente en diferentes niveles: internacional, nacional o central, local, regional y de origen de los datos. A nivel nacional, la estadística hospitalaria nacional permite hacer comparaciones con otros hospitales que conforman la red hospitalaria del país. El valor fundamental de la estadística hospitalaria reside en su función como estadística de recursos para la planificación de la salud (Campos Santillan, 1966). 


\section{Aspectos conceptuales}

Se describe a continuación algunos conceptos que son desarrollados en la investigación:

\section{Defunción}

De acuerdo a la Real Academia Española, procede del latín defunctio, -onis, muerte de una persona (Española, 2014). Es la desaparición permanente de todo signo de vida, cualquiera sea el tiempo transcurrido desde el nacimiento con vida (cesación postnatal de las funciones vitales sin posibilidad de resucitar). Esta definición excluye las defunciones fetales (http://palma.ine.cl/demografia/ menu/glosario.pdf, Glosario).

\section{Causa Básica de muerte de la defunción}

Enfermedad o lesión que inició la cadena de acontecimientos patológicos que condujeron directamente a la muerte o las circunstancias del accidente o violencia que produjo la lesión fatal (http://palma.ine.cl/demografia/ menu/glosario.pdf, Glosario).

\section{$\longrightarrow$ Clasificación de Enfermedades 10}

Se puede definir como: "Sistema de categorías a las cuales se les asignan entidades morbosas de acuerdo con criterios establecidos " (OPS, Clasificación Estadística Internacional de Enfermedades y Problemas relacionados con la Salud, 1992).

\section{Mortalidad proporcional por causas}

Es el riesgo de morir de la población en un período dado por determinada causa, entendiéndose ésta por enfermedad, discapacidad o violencia accidental o intencional (Cuba, 2010). Por definición es el número de muertes durante un período específico de tiempo en un lugar (numerador) sobre Total de muertes en ese período y en ese lugar (denominador) x 100 (Cárcamo, 2016). Cuando se usa este indicador epidemiológico se debe tener, por lo menos, información confiable por la causa de interés en la población en estudio (Bergonzoli, 1999).

\section{Discusión de resultados}

Se describen a continuación los resultados de la investigación en las siguientes variables: sexo, edad en datos no agrupados, describiendo medidas de tendencia central y de desviación, causa de muerte básica de acuerdo a clasificación, tasa de mortalidad proporcional por causas.

\subsection{Mortalidad hospitalaria proporcional por sexo, años 2014, 2015 y 2016}

La mortalidad se manifiesta en los 3 años de forma contundente en el sexo femenino, el porcentaje de muerte, aumentó en el año 2015, para posteriormente disminuir en el año 2016. En el caso de los hombres, se observa una disminución en los tres años. La razón entre ambos sexos, es de 2:1 en los tres años a favor del sexo masculino, los que sobreviven el doble que las mujeres. (Datos en cuadro No. 1). En relación a lo anterior, los datos que se muestran en el documento de los perfiles de país de la OMS en el año 2014, establecen que en la distribución de la mortalidad por sexo por cáncer, el número de mujeres que murieron fueron 2,700 y los hombres, 2,400. El observar la distribución, el $46.5 \%$ de las causas en las mujeres se tipificaron como "otras", el segundo lugar, lo ocupa el cáncer cervicouterino con el $15.2 \%$; el de estómago con el 14.2\%; en el tercer lugar, el de hígado con el $10.9 \%$ y el de mama, ocupa el cuarto lugar, con el 8.9\% (OMS, Perfiles de cáncer de Honduras, 2014). En relación a los datos de ese mismo año, la mortalidad por sexo, en el Hospital San Felipe, en relación a los años de vida potencialmente perdidos, demuestran que las mujeres suman 1,054 AVPP en relación a los hombres que suman 491 AVPP por causa de los tumores, que ocupan el primer lugar, como causa de muerte (Garay Carolina, 2015). De acuerdo a los datos señalados, la magnitud de la mortalidad en mujeres por los tumores, es importante y representa un señalamiento para las políticas públicas en salud.

Cuadro No.1. Mortalidad hospitalaria proporcional por sexo, años 2014, 2015 y 2016

\begin{tabular}{|c|c|c|}
\hline Año & Hombre & Mujer \\
\hline 2014 & 37.5 & 62.5 \\
\hline 2015 & 35.6 & 64.4 \\
\hline 2016 & 37.18 & 62.82 \\
\hline
\end{tabular}

Fuente: expedientes clínicos de pacientes. Dpto. de Estadística de HSF.

\subsection{Mortalidad hospitalaria proporcional por edad en los años 2014, 2015 y 2016}

En relación a los datos no agrupados, en el año 2014, la media de edad, es de 55.52, la mediana de 57.5 años, la moda es de 62. La desviación estándar es de 18.8 y la varianza es de 355.7, el rango es de 94 años. En relación a las medidas de dispersión, la desviación estándar evidencia la variación existente entre los elementos del grupo de análisis. En cuanto a la varianza, ésta muestra la variación cuadrática de los datos con respecto a la me- 
dia. El rango establece que tan dispersa se encuentra la distribución tomando la diferencia de los valores máximos y mínimos. Los datos reflejan la dispersión de los datos de la edad, tomando en cuenta que la edad mínima para este año es de 1 año y el valor máximo es de 95 años.

Para el año 2015, la media es de 57.45, la mediana de 61 , la moda de 62. La desviación estándar es de 20.2 y la varianza de 409.3, el rango es de 96 años. Las medidas de dispersión establecen a través de la desviación estándar la variación que existe entre los datos de edad de cada paciente y la varianza, establece la variación cuadrática con respecto a la media. El rango señala la diferencia que existe del valor mínimo (1 año) y el valor máximo (97 años).

En el año 2016, la media de edad es de 57.29, la mediana de 61 , la moda mínima de 59. La desviación estándar es de 19.4 y la varianza de 377.9. El rango es de 93 años (Cuadro No. 2).Para este año, las medidas de dispersión muestran la dispersión existente tomando en cuenta que el valor mínimo de edad es de 1 año y el máximo de 94 años. Por lo general, la mortalidad es alta o relativamente alta al inicio de la vida, en especial durante los primeros cinco años de vida. Durante los cinco a diez años de edad alcanza los niveles más bajos, dependiendo del nivel de la mortalidad del país. A partir de los 20 años comienza a aumentar levemente la mortalidad hasta los 40 o 50 años y a partir de estas edades se incrementa las tasas más rápidamente para alcanzar nuevamente niveles más elevados en las edades más avanzadas (Mazzeo, 2014). La fuerte relación entre la edad y la mortalidad hace que sea fundamental conocer la estructura de edades del fenómeno (http://www.ugr.es/ fabad/MORTALIDAD_TEORIA.pdf)

Cuadro No.2. Mortalidad hospitalaria por edad en los años 2014, 2015 y 2016

\begin{tabular}{|c|c|c|c|c|c|c|}
\hline Años & Media & Mediana & Moda & $\begin{array}{c}\text { Desv } \\
\text { est }\end{array}$ & $\begin{array}{c}\text { Va- } \\
\text { rianza }\end{array}$ & Rango \\
\hline 2014 & 55.52 & 57.5 & 62 & 18.8 & 355.7 & 94 \\
\hline 2015 & 57.45 & 61 & 62 & 20.2 & 409.3 & 96 \\
\hline 2016 & 57.29 & 61 & 59 & 19.4 & 377.9 & 93 \\
\hline
\end{tabular}

Fuente: datos de expedientes clínicos de pacientes. Dpto. de Estadistica de HSF.

\subsection{Mortalidad proporcional por causa}

En los tres años observados, la primera causa de muerte es el cáncer, cuya proporción va en aumento en cada año. La septicemia, ha ido en disminución y en los dos últimos años observados, se encuentra estable.

Las enfermedades del aparato digestivo, presentaron aumento en el año 2015, pero en el año 2016, cifra bajó más que en el año 2014. Las enfermedades del aparato respiratorio, han mostrado una disminución en los tres años. Las enfermedades del aparato circulatorio, bajaron a casi la mitad de la cifra inicial que parte del 2014 y nuevamente en el año 2016, vuelve a ascender sin alcanzar la proporción del año 2014.

Es a partir de este indicador que se logra establecer: la razón entre la primera causa (cáncer) y la penúltima (enfermedades del aparato circulatorio), para el año 2014, es de 8:1; para el año 2015, es de $16: 1$ y para el año 2016, es de 16:1 (Cuadro No.3).

Cuadro No.3. Mortalidad proporcional por causa de años 2014, 2015 y 2016

\begin{tabular}{|c|c|c|c|c|c|c|c|}
\hline \multirow{2}{*}{ Causa de muerte } & \multirow{2}{*}{ CIE-10* } & \multicolumn{2}{|c|}{2014} & \multicolumn{2}{|c|}{2015} & \multicolumn{2}{|c|}{2016} \\
\hline & & No. Absoluto & Proporción & No. absoluto & Proporción & No. Absoluto & Proporción \\
\hline Cáncer & C07X-C859 & 73 & 60.83 & 118 & 66.6 & 114 & 73.07 \\
\hline Septicemia & A 419 & 16 & 13.33 & 17 & 9.604 & 15 & 9.615 \\
\hline $\begin{array}{l}\text { Enfermedad del aparato } \\
\text { digestivo }\end{array}$ & K00-K93 & 7 & 5.83 & 17 & 9.604 & 7 & 4.487 \\
\hline $\begin{array}{l}\text { Enfermedad del aparato } \\
\text { respiratorio }\end{array}$ & J00-J99 & 7 & 5.83 & 7 & 3.95 & 4 & 2.564 \\
\hline $\begin{array}{l}\text { Enfermerdad del aparato } \\
\text { circulatorio }\end{array}$ & I00-I99 & 9 & 7.5 & 7 & 3.95 & 7 & 4.487 \\
\hline $\begin{array}{l}\text { Causas de mortalidad } \\
\text { mal definidas y descono- } \\
\text { cidas en niños de un año }\end{array}$ & R95-R99 & 4 & 3.33 & 4 & 2.25 & 5 & 3.2 \\
\hline Diabetes & E110-E129 & 4 & 3.33 & 7 & 3.95 & 4 & 2.56 \\
\hline Total & 120 & & & 177 & & 156 & \\
\hline
\end{tabular}

Fuente: datos de expedientes clínicos de pacientes. Depto. de Estadística de HSF. *Clasificación Internacional de Enfermedades 10 


\section{Conclusión}

La mortalidad hospitalaria ha sido propuesta como un indicador de calidad asistencial. En el mundo, las prevalencias de enfermedades en los ingresos hospitalarios no coinciden necesariamente con las de causas de mortalidad; este es el caso de los establecimientos que tienen maternidad, donde la mayor causa de ingresos es por nacimientos e influye poco sobre la mortalidad hospitalaria; por el contrario, las neoplasias son una de las causas prevalente de mortalidad, sobre todo en los países desarrollados (Revista, 2013).

Se ha establecido que: "...Todo es susceptible de ser medido en este mundo...". El asunto radica en cómo medirlo y con qué medirlo. Cuando se trata del área de la salud, se pretende que el método con el cual se mide, sea confiable y que los resultados de las mediciones permitan evaluar si se ha logrado o no, algún avance de importancia y que los resultados visualicen algunos avances e impacto en las políticas de salud (De la Fuente \& Tapia, 2001).

El interés de cómo medir la salud, está relacionado con la evaluación de la atención de la salud. Medidas de estructura, proceso y resultado han sido utilizadas con este fin, dependiendo del contexto de la evaluación. La mortalidad es un indicador de daño y su "ventaja" es que la muerte es un evento único que generalmente, se registra (Schaepfer \& Infante, 1990).

Los indicadores de salud a nivel de hospitales, representan medidas-resumen que capturan información relevante sobre distintos atributos y dimensiones del estado de salud y del desempeño del sistema de salud. Generados de manera regular y manejados dentro de un sistema de información dinámica, los indicadores de salud constituyen una herramienta fundamental para los tomadores de decisiones en todos los niveles de gestión (Salud S. d., 2008) En relación a los datos observados en el Hospital San Felipe, situado en la capital de Honduras, en los tres años de 2014 al 2016, denotan que la primera causa de muerte, es cáncer y otras enfermedades no transmisibles; el sexo que presenta mayor número de defunciones es el femenino. De acuerdo a la nota descriptiva de la OMS, sobre salud de la mujer, las enfermedades no transmisibles impactan a partir de la edad de los 6 años, sobre todo el cáncer y las enfermedades cardiovasculares, con independencia del desarrollo económico del país en el que viven.

En el caso que nos ocupa, la media de edad de las muertes, se encuentran en los 57 y la mediana en los 61 , los que estaría acorde con las cifras establecidas por este organismo internacional, añadiendo el hecho de que las enfermedades cardiovasculares son responsables de la mortalidad en el $46 \%$ de estas mujeres en estos grupos de edad, en tanto que el cáncer y las afecciones respiratorias, crónicas, sobre todo, la enfermedad pulmonar obstructiva crónica (EPOC), ocuparían el segundo y el tercer lugar.

Muchos de los problemas de salud que padecen las mujeres en etapas más avanzadas de la vida se deben a la exposición a diversos factores de riesgo durante la adolescencia y etapas anteriores de la edad adulta, como el tabaquismo, sedentarismo y mala alimentación (OMS, Salud de la mujer. Nota descriptiva No.334, 2013)

En la casuística presentada, es el cáncer, el que ocupa el primer lugar. Al respecto, la OMS establece que los tipos más frecuentes de cáncer son diferentes en los hombres y en las mujeres. Aproximadamente un $30 \%$ de las muertes se deben a cinco factores de riesgo comportamentales y alimentarios: índice de masa corporal elevado, consumo insuficiente de frutas y verduras, falta de actividad física y consumo de tabaco y alcohol, por lo tanto, pueden prevenirse (OMS, Datos y cifras sobre el cáncer , 2014).

Es necesario añadir que el $70 \%$ de las muertes por cáncer en el año 2012, ocurrieron en África, Asia, América Central y Sudamérica. Se prevé que los casos anuales de cáncer aumentarán de 14 millones en 2012 a 2022 en las próximas dos décadas. (OMS, Datos y cifras sobre el cáncer , 2014).

En Honduras, en el año 2014, se atendieron 829 casos de cáncer (mama, cuello uterino, próstata, gástrico y pulmón) en los hospitales de la Secretaría de Salud (Salud S. d., 2015).

La segunda causa de muerte, es la septicemia. La casuística internacional establece que un elevado porcentaje de las muertes hospitalarias se producen en pacientes con septicemia y una elevada proporción de estas muertes se producen en "sepsis no críticas". La epidemiología de la septicemia no se conoce de forma completa.

Uno de los principales problemas es el "diagnóstico" poco utilizado, que se codifica pocas veces en las bases de datos administrativas de los hospitales (se suele codificar únicamente la infección que produce la sepsis, lo que hace difícil cuantificar su impacto real (Palencia Herrejón, 2014)

Los datos muestran la mortalidad por afecciones en niños menores de un año y en ese sentido, estudios realizados en Uruguay muestran que las causas más frecuentes de muerte fueron de origen infeccioso (65\%), seguidas por 
las anomalías congénitas (13\%) (Ferrari, Ferreira, De Leonardis, Fernández, \& Imbriaco, 2002).

En estudios realizados en Argentina, las causas de mortalidad en menores de un año, están relacionadas con desnutrición o enfermedad infecciosa, así como las malformaciones congénitas. Para el año 2006, fue de 12.9\%. La mortalidad infantil, es un fenómeno en el cual la enfermedad y la muerte de los niños menores de un año se presentan como un fenómeno biológico en un contexto social (Lomuto, 2006).

Datos proporcionados por la OMS en el año 2015, señalan que la mortalidad en menores de un año en la Región de las Américas es de 13 por 1000 nacidos vivos y la primera causa que la produce es prematuridad, la segunda causa las anomalías congénitas. El bajo peso al nacer no se considera una causa directa de muerte pero es un factor predisponente (OPS \& OMS, Informe final sobre los Objetivos de Desarrollo del Milenio relacionados con la salud en la Región de las Américas, 2017)

La utilidad de las estadísticas hospitalarias como apoyo al proceso de planificación, fortalecería todo proceso de planificación que, a su vez, fortalecería las propuestas económicas para programar e instrumentar estos servicios. La especificidad de los datos presentados, tras el hecho de ser una revisión de tres años, apoyará con mayor énfasis a los servicios de salud que se encuentran evidenciados en el hospital, sobre todo, porque se trata de un hospital de referencia nacional, que atiende a pacientes de todo el país.

La utilización de los recursos de estadísticas hospitalarias establece la demanda a través de la demanda registrada, que es en función de la demanda real que, a su vez, es función de la demanda potencial. Los estudios de mortalidad en hospitales, son escasos (García Ortega, Almenara Barrios, \& García Ortega, 1997), razón por la cual, se deben continuar desarrollando varias modelos de investigación que incluyan aspectos de ajustes de riesgo con lo cual supone abrir otras puertas de investigación para la mejora de los servicios y sobre el fortalecimiento de varios aspectos en la atención hospitalaria, como ser la garantía de la calidad de la misma, el Hospital San Felipe, debe realizar esa planificación considerando estos aspectos (OMS, Estadísticas hospitalaria sobre las causas de defunción: qué hacer con ellas?, 2014).

\section{Bibliografía}

Bergonzoli, G. (1999). Cuán válida es la razón de mortalidad proporcional? Cali: Colombia Médica, vol.30, No.3, 1999, pp. 132-136.

Campos Santillan, T. (1966). Conceptos Modernos en estadística hospitalaria. OPS.

Cárcamo, M. (2016). Conceptos básicos de las medidas y los indicadores de la Epidemiología. Santiago: Universidad de los Andes.

Cuba, M. d. (2010). Indicadores Básicos para el análisis del estado de salud de la población. Habana.

De la Fuente, J. R., \& Tapia, R. (2001). La Medición en Salud a través de Indicadores. México, D.F: UNAM.

Delgado R, M., Sillero, M., \& Galvez, R. (1994). Estudios de Mortalidad Proporcional: criterios de elección de los grupos participantes. Madrid.

W Española, R. (2014). Diccionario de la lengua española/ Edición del Tricentenario.

Ferrari, A. M., Ferreira, A., De Leonardis, D., Fernández, A., \& Imbriaco, J. (2002). Mortalidad hospitalaria en un hospital pediátrico de referencia nacional:Centro Hospitalaria Pereira Rosell. Montevideo: Rev.Med.Urug. vol 18. no.1.

Garay Carolina, R. M. (2015). Mortalidad Hospitalaria y Años de Vida Potencialmente perdidos en Hospital San Felipe. Tegucigalpa.

M García Ortega, C., Almenara Barrios, J., \& García Ortega, J. (1997). Tasas específicas de mortalidad en el Hospital de Algeciras durante el período 1995-1996. Rev Esp Salud Pública 1997; 71:305-315.

http://palma.ine.cl/demografia/menu/glosario.pdf. (s.f.). Glosario.

http://palma.ine.cl/demografia/menu/glosario.pdf. (s.f.). Glosario.

http://publications.paho.org/spanish/PC+629+Cap_2. pdf. (s.f.).

http://www.ugr.es/ fabad/MORTALIDAD_TEORIA.pdf. (s.f.).

Lomuto, C. (2006). Mortalidad infantil y neonatal.

Mazzeo, V. (2014). Unidad de Mortalidad. Demografía Social. Serie Apuntes de clases No.1. 
W OMS. (2013). Salud de la mujer. Nota descriptiva No.334. Ginebra.

OMS. (2014). Datos y cifras sobre el cáncer. Ginebra.

OMS. (2014). Estadísticas hospitalaria sobre las causas de defunción: qué hacer con ellas? Ginebra.

OMS. (2014). Perfiles de cáncer de Honduras. Ginebra.

OMS. (2016). Reducción de la mortalidad en recién nacidos. Nota descriptiva No.333.

OPS. (1992). Clasificación Estadística Internacional de Enfermedades y Problemas relacionados con la Salud. Ginebra.

W OPS. (2004). Evaluación decenal de la Iniciativa Regional de Datos Básicos de Salud. Washington: Boletín Epidemiológico.

OPS, \& OMS. (2017). Informe final sobre los Objetivos de Desarrollo del Milenio relacionados con la salud en la Región de las Américas. Washington.

Palencia Herrejón, E. (2014). Contribución de la sepsis a la mortalidad hospitalaria. IntraMed.

W Perú, M. d. (2013). Indicadores de gestión y evaluación hospitalaria para hospitales, institutos y Diresa.

Revista, M. (2013). Análisis de la mortalidad hospitalaria. Buenos Aíres: ISSN 0025-7680.

W Salud, S. d. (2008). Manual de Indicadores de Servicios de Salud.

Salud, S. d. (2015). Memoria Anual 2014. Tegucigalpa.

W Schaepfer, L., \& Infante, C. (1990). La meidición de salud: Perspectivas teórica y metodológicas. Cuernavaca: Salud Pública de México, vol.32, núm 2, marzo-abril, 1990, p.p 141-155.

Segura, A. (1989). Contribución del médico al conocimiento de los problemas de salud de la comunidad: la certificación de las causas de muerte. Barcelona. 